\title{
A FURTHER NECESSARY AND SUFFICIENT CONDITION FOR STRONG CONVERGENCE OF NONLINEAR CONTRACTION SEMIGROUPS AND OF ITERATIVE METHODS FOR ACCRETIVE OPERATORS IN BANACH SPACES
}

\author{
by ZONG-BEN XU, YAO-LIN JIANG and G. F. ROACH \\ (Received 15th May 1992)
}

Let $A$ be a quasi-accretive operator defined in a uniformly smooth Banach space. We present a necessary and sufficient condition for the strong convergence of the semigroups generated by $-A$ and of the steepest descent methods to a zero of $A$.

1991 Mathematics subject classification: 47H06, 47H15, 65J15.

\section{Introduction}

The purpose of this paper is to present a necessary and sufficient condition for the strong convergence of the semigroup generated by a $m$-accretive operator and of the steepest descent approximation process.

$$
x_{n+1}=x_{n}-t_{n} A x_{n}, \quad t_{n} \in R^{+}=(0, \infty), \quad\left\{t_{n}\right\} \notin l^{1}
$$

to a zero of a quasi-accretive operator $A$ in Banach spaces.

Let $X^{*}$ be the dual space of a real Banach space $X$ and $J: X \rightarrow 2^{X^{*}}$ be the normalized duality mapping defined by

$$
J x=\left\{x^{*} \in X^{*}:\left\langle x^{*}, x\right\rangle=\left\|x^{*}\right\|\|x\|,\left\|x^{*}\right\|=\|x\|\right\} \quad \forall x \in X
$$

where $\langle.,$.$\rangle denotes the generalized duality pairing. An operator A$ with domain $D(A)$, range $R(A)$ and kernel $N(A)$ is said to be accretive if, for every $x, y \in D(A)$, there is a selection $j(x-y) \in J(x-y)$ such that $\langle A x-A y, j(x-y)\rangle \geqq 0$. If $N(A) \neq 0$ and this inequality holds for any $x \in D(A)$ but $y \in N(A)$, the corresponding operator $A$ is said to be quasi-accretive. An accretive operator $A$ is $m$-accretive if $R(I+r A)=X$ for all $r>0$.

Such operators have beem extensively studied and applied by various authors (see, e.g. [1]-[8], [10]-[14]). The interest and importance of such operators stems mainly from the fact that many physically significant problems can be modelled in terms of an initial value problem of the form 


$$
\begin{aligned}
& x^{\prime}(t)+A x(t)=0 \\
& x(0)=x_{0}
\end{aligned}
$$

where $A$ is an accretive operator in an appropriate Banach space. In this case, a zero of $A$ corresponds to an equilibrium point of the system (1.2).

When $A$ is a $m$-accretive operator in a reflexive Banach space, it is known [3, p. 118] that $-A$ generates a semigroup $S$ of nonlinear contraction on $c l(D(A))$, the closure of $D(A)$, and $S(t) x_{0}$ for any $x_{0} \in D(A)$ is a strong solution of (1.2). Therefore, the asymptotic behaviour of the system (1.2) is reflected by the convergence of the semigroup $S(t)$ as well as the process (1.1), which can be viewed as a discrete approximation of $S(t)$, as $t$ goes to infinity. In this line, several sufficient conditions are known. See, for instance, O. Nevanlinna and S. Reich [10], R. E. Bruck and S. Reich [7], A. Pazy [11] and the references mentioned there. In this paper we would like to characterize the necessary and sufficient conditions for the strong convergence of $S(t) x_{0}$ and the process (1.1) in Banach spaces. In [16], Z. B. Xu and G. F. Roach considered this problem for certain special cases of the operators that possess the so-called property $(I)$. We shall extend these previously established results. In particular, our main theorems (Theorems 1 and 2) will unify and generalize all of the corresponding results established in [6]-[8], [10]$[11],[13]-[14]$ and $[16]$.

\section{Preliminaries}

Given a quasi-accretive operator $A$ in a Banach space $X$, we let $N_{0}(A)$ be a proximal, closed and convex subset of $N(A), P_{0}$ be an arbitrary selection of the nearest point mapping from $X$ onto $N_{0}(A)$ and $J_{0}\left(x-P_{0} x\right)$ be an element in $J\left(x-P_{0} x\right)$ that satisfies $\left\langle J_{0}\left(x-P_{0} x\right), P_{0} x-y\right\rangle \geqq 0$ for all $y \in N_{0}(A)$. With this notation, we introduce the following:

Definition 1. The operator $A$ is said to satisfy the condition $(J)$ if $\left\langle A x, J_{0}\left(x-P_{0} x\right)\right\rangle=0$ implies $x \in N(A)$.

Definition 2. A sequence $\left\{x_{n}\right\}$ (an abstract function $u(t)$ ) in $X$ is said to be hyperstrongly convergent if it is strongly convergent, the limit $m=\lim _{n \rightarrow \infty}\left\|x_{n}-P_{0} x_{n}\right\|$ $\left(\lim _{t \rightarrow \infty}\left\|u(t)-P_{0} u(t)\right\|\right)$ exists and either $m=0$ or $\left.\left\{\left\|x_{n+1}-x_{n}\right\|\right\} \in l^{1}(\| d u / d t) \| \in L^{1}(0, \infty)\right)$.

The following provides us with a series of examples of operators satisfying the condition $(J)$.

(i) $A$ satisfies the condition (I) introduced in [16], that is, for any $p \in N(A)$ and any $j(x-p) \in J(x-p)$, the equality $\langle A x, j(x-p)\rangle=0$ holds if and only if $x \in N(A)$. In this setting, it is seen that $A$ satisfies the condition $(J)$ with $N_{0}(A)=\{p\}$ for any $p \in N(A)$. Furthermore, either strongly quasi-accretive operators or operators of the form $A=\alpha(I-T)$ with a constant $\alpha$ and a nonexpansive mapping $T$ defined in a uniformly convex Banach space (cf. [16, Th. 2]) also satisfy $(J)$. 
(ii) $A$ satisfies the convergence condition introduced by Nevanlinna and Reich in [10]: if $X$ is uniformly convex and $A$ is $m$-accretive, then $x_{n} \in D(A),\left\|x_{n}\right\| \leqq C,\left\|A x_{n}\right\| \leqq C$ and $\lim _{n \rightarrow \infty}\left\langle A x_{n}, J\left(x_{n}-P x_{n}\right)\right\rangle=0$ imply that $\liminf _{n \rightarrow \infty}\left\|x_{n}-P x_{n}\right\|=0$, where $P: X \rightarrow N(A)$ is the nearest point mapping. From [7] and [10], it is clear that such operators satisfy the condition $(J)$ with $N_{0}(A)=N(A)$ and, particularly, they include those operators such that $N(A)=\{z\}$ and $0 \in \operatorname{int}(A z)$.

(iii) $A$ is quasi-accretive and demi-positive in the sense of Bruck [4]: $A$ is a quasiaccretive operator such that for any $x_{n} \in D(A)$ and for an $p \in N(A)$, if $x_{n} \stackrel{w}{\longrightarrow} x,\left\{A x_{n}\right\}$ is bounded and $\left\langle A x, J\left(x_{n}-p\right)\right\rangle \rightarrow 0(n \rightarrow \infty)$, then $x \in N(A)$. It is seen that a demi-positive operator satisfies the condition $(J)$ with $N_{0}(A)=\{p\}$. If $X$ is uniformly convex and smooth, any accretive operators that have zero sets with nonempty interiors are demi-positive.

(iv) A satisfies Bruck's condition (see [6]): if $x_{n} \in D(A)$ such that $x_{n} \stackrel{w}{\rightarrow} x$ then $\lim _{n \rightarrow \infty}\left\langle A x_{n}-A y, x_{n}-y\right\rangle=0$ implies $A y=A x$. The class of operators satisfying this condition in Hilbert spaces, for instance, includes those maximal monotone operators of subdifferentials, or more generally $\sigma$-angle-bounded operators and Yosida approximation $A_{\lambda}$ of a maximal monotone operator (cf. $[6, \mathrm{p} .15]$ ).

We shall establish our main results by using certain special geometric aspects of Banach spaces. Recall that a Banach space is said to be uniformly smooth if the modulus of smoothness of $X$, defined by

$$
\rho_{X}(\tau)=\frac{1}{2} \sup \{\|x+y\|+\|x-y\|-2: \quad\|x\|=1,\|y\| \leqq \tau\}
$$

satisfies $\lim _{\tau \rightarrow 0} \rho_{X}(\tau) / \tau=0$. The following properties of $\rho_{X}$ will be used in the sequel:

(p1) $\rho_{X}(0)=0, \rho_{X}(\tau) \leqq \tau$;

(p2) $\rho_{X}(\tau)$ is continuous, convex and nondecreasing;

(p3) $\rho_{X}(\tau) / \tau$ is nondecreasing;

(p4) There is a constant $B>0$ such that $\rho_{X}(\eta) / \eta^{2} \leqq B \rho_{X}(\tau) / \tau^{2}$ for any $\eta \geqq \tau>0$.

We also need the following specialization of $[15, \mathrm{Th} .2]$ :

Lemma 1 [16]. Let $X$ be a uniformly smooth Banach space with its modulus of smoothness $\rho_{X}(\tau)$. Then there exist positive constants $K$ and $C$ such that

$$
\|x+y\|^{2} \leqq\|x\|^{2}+2\langle J x, y\rangle+K \max \left\{\|x\|+\|y\|, \frac{1}{2} C\right\} \rho_{X}(\|y\|), \quad \forall x, y \in X .
$$

\section{The steepest descent approximation}

With the notation fixed as above, we now prove the following theorem.

Theorem 1. Let $X$ be $a$ uniformly smooth Banach space and $A: X \rightarrow X$ be $a$ quasi-accretive, demi-closed operator which satisfies the condition $(J)$ and

$$
\sup \left\{\|A x\|:\left\|x-P_{0} x\right\| \leqq C\right\}<+\infty, \quad \forall C>0 .
$$


Then, for any $x_{0} \in X$ there is a positive real number $T\left(x_{0}\right)$ such that the sequence $\left\{x_{n}\right\}$ defined by the steepest descent approximation process (1.1) with

$$
t_{n} \leqq T\left(x_{0}\right), \quad n=0,1, \ldots, \text { and }\left\{\rho_{X}\left(t_{n}\right)\right\} \in l^{1}
$$

converges hyperstrongly to an element $x^{*}$ in $N(A)$ if and only if there is a nondecreasing function $\psi: R^{+} \rightarrow R^{+}$such that

$$
\left\langle A x_{n}, J\left(x_{n}-P_{0} x_{n}\right)\right\rangle \geqq \psi\left(\left\|x_{n}-P_{0} x_{n}\right\|\right)\left\|A x_{n}\right\|, \quad n=0,1, \ldots
$$

where $\psi(0)=0, \psi(t)$ is strictly increasing in $t \geqq t^{*}$ with

$$
t^{*}=\left\{\begin{array}{l}
\lim _{n \rightarrow \infty}\left\|x_{n}-P_{0} x_{n}\right\|, \text { if }\left\{\left\|x_{n+1}-x_{n}\right\|\right\} \in l^{1} \\
0, \quad \text { otherwise }
\end{array}\right.
$$

Proof. “ $\Rightarrow "$ Let $\quad M=\sup \left\{\left\|x_{n}-P_{0} x_{n}\right\|: n \geqq 0\right\}, \quad N=\sup \left\{\left\|A x_{n}\right\|: n \geqq 0\right\} \quad$ and $m=$ $\lim _{n \rightarrow \infty}\left\|x_{n}-P_{0} x_{n}\right\|$. If $M=0$ or $N=0$, then $x_{n}=x^{*}$ for some $x^{*} \in N(A)$ and hence (3.3) follows trivially; If $M=m$, then (3.3) follows also directly provided we define $\psi(t)$ to be any strictly increasing function in $t>t^{*}$ but $\psi(t)=0$ for every $t \in\left[0, t^{*}\right]$. Suppose $M>0$, $N>0$ and $M>m$. Then for any $t \in(0, M)$ we define

$$
C_{t}=\left\{n \in N:\left\|x_{n}-P_{0} x_{n}\right\|>t\right\}
$$

and

$$
f(t)=\inf \left\{\left\langle A x_{n}, J\left(x_{n}-P_{0} x_{n}\right)\right\rangle / N: n \in C_{t}\right\}
$$

Clearly $f(t)$ is nonnegative and nondecreasing. We now prove that $f(t)>0$ for any $t \in(m, M)$. Assume this is not the case, namely, that there is a $t_{0} \in(m, M)$ such that $f\left(t_{0}\right)=0$. Then a subsequence $\left\{n_{i}\right\}$ in $C_{t_{0}}$ exists so that

$$
\left\|x_{n_{i}}-P_{0} x_{n_{i}}\right\| \geqq t_{0}>m
$$

and

$$
\left\langle A x_{n_{i}}, J\left(x_{n_{i}}-P_{0} x_{n_{i}}\right)\right\rangle / N \rightarrow 0 \quad(i \rightarrow \infty) .
$$

Since the limit $m=\lim _{n \rightarrow \infty}\left\|x_{n}-P_{0} x_{n}\right\|$ exists, (3.5) implies that $\left\{\left\|x_{n_{i}}-P_{0} x_{n_{i}}\right\|\right\}$ is finitely circulative, that is, there exists an $x_{n_{0}} \in\left\{x_{n}\right\}$ and a subsequence $\left\{x_{n_{i}}\right\} \subset\left\{x_{n_{i}}\right\}$ such that $x_{n_{i j}}=x_{n_{0}}$ for any $j \geqq 1$. $x_{n_{0}}$ must be an element in $N(A)$ because (3.6) implies $\left\langle A x_{n_{0}}, J_{0}\left(x_{n_{0}}-P_{0} x_{n_{0}}\right)\right\rangle=0$ and $A$ satisfies the condition $(J)$. Thus, by the definition of the process (1.1), we have $x_{n}=x_{n_{0}}$ for any $n \geqq n_{0}$. Since $x_{n}$ is known to converge to $x^{*} \in N(A)$, it in turn follows that $x_{n_{0}}=x^{*}$. Therefore we deduce from (3.5) that $\left\|x^{*}-P_{0} x^{*}\right\| \geqq t_{n}>m$. But, on the other hand, we have 


$$
\left\|x^{*}-P_{0} x^{*}\right\| \leqq\left\|x^{*}-P_{0} x_{n}\right\| \leqq\left\|x^{*}-x_{n}\right\|+\left\|x_{n}-P_{0} x_{n}\right\|
$$

This yields $\left\|x^{*}-P_{0} x^{*}\right\| \leqq \lim _{n \rightarrow \infty}\left\|x_{n}-P_{0} x_{n}\right\|=m$, which is a contradiction. Thus, $f(t)>0$ for any $t \in(m, M)$. Moreover, by the definition of $t^{*}$, if $\left\{\left\|x_{n+1}-x_{n}\right\|\right\} \in l^{1}$ then $t^{*}=m$ and if $\left\{\left\|x_{n+1}-x_{n}\right\|\right\} \notin l^{1}$, by the hyperstrong convergence of $\left\{x_{n}\right\}$ we also have $m=0=t^{*}$, we conclude that $f(t)>0$ for any $t \in\left(t^{*}, M\right)$.

We extend the domain of $f$ to $R^{+}$by defining $f(0)=0, f(t)=\sup \{f(s): s<M\}$ for every $t \geqq M$ and $f(t)=\inf \{f(s): s<M\}$ for any $t \in(0, m]$. Then, with the function $\psi$ defined by $\psi(t)=(1+t)^{-1} t f(t)$, it is seen that $\psi(0)=0, \psi(t)$ is strictly increasing in $t \geqq t^{*}$, which fulfills the inequality (3.3). This completes the proof of the necessity.

" $\Leftarrow$ ". Suppose the inequality (3.3) is satisfied for a function $\psi$ that possesses the mentioned properties. We prove that there is a $T\left(x_{0}\right)$ such that the process (1.1) with $t_{n}$ chosen as in (3.2) converges hyperstrongly to $x^{*}$, an element in $N(A)$. To this end, we assume $x_{0} \notin N(A)$ and let $\mu$ be a positive real number such that

$$
\left\|x_{0}-P_{0} x_{0}\right\| \leqq 2 \mu\left\|A x_{0}\right\| \text { and } \psi\left(\mu\left\|A x_{0}\right\|\right)>0 .
$$

Let

$$
\begin{gathered}
M\left(x_{0}\right)=\sup \left\{\|A x\|:\left\|x-P_{0} x\right\| \leqq 2 \mu\left\|A x_{0}\right\|\right\} \\
\beta=\sup \left\{b: \rho_{X}\left(b M\left(x_{0}\right)\right) / b \leqq \frac{2 \psi\left(\mu\left\|A x_{0}\right\|\right)\left\|A x_{0}\right\|}{K\left(3 \mu\left\|A x_{0}\right\|+C / 2\right)}\right\} \\
T\left(x_{0}\right)=\min \left\{\beta, \mu\left\|A x_{0}\right\| / M\left(x_{0}\right)\right\}
\end{gathered}
$$

where the constants $K$ and $C$ are the same as in Lemma 1. We proceed by the following steps:

Step 1. We prove that the sequences $\left\{x_{n}-P_{0} x_{n}\right\}$ are bounded and in particular,

$$
\left\|x_{n}-P_{0} x_{n}\right\| \leqq 2 \mu\left\|A x_{0}\right\|, \quad n=0,1, \ldots
$$

This is trivially true for $n=0$. Assume this holds for an integer $n>0$. Then we have two possible cases: either $\left\|x_{n}-P_{0} x_{n}\right\| \leqq \mu\left\|A x_{0}\right\|$ or $\left\|x_{n}-P_{0} x_{n}\right\|>\mu\left\|A x_{0}\right\|$. In the first case, by (3.7)-(3.10) and (1.1) we have

$$
\begin{aligned}
&\left\|x_{n+1}-P_{0} x_{n+1}\right\| \leqq\left\|x_{n+1}-P_{0} x_{n}\right\| \leqq\left\|x_{n}-P_{0} x_{n}\right\|+t_{n}\left\|A x_{n}\right\| \\
& \leqq \mu\left\|A x_{0}\right\|+T\left(x_{0}\right) M\left(x_{0}\right) \leqq 2 \mu\left\|A x_{0}\right\|
\end{aligned}
$$

which establishes (3.11) directly. In the second case, by using Lemma 1 and (3.7) $-(3.10)$, we obtain 


$$
\begin{aligned}
\left\|x_{n+1}-P_{0} x_{n+1}\right\|^{2} \leqq & \left\|x_{n+1}-P_{0} x_{n}\right\|^{2} \\
\leqq & \left\|x_{n}-P_{0} x_{n}\right\|^{2}-2 t_{n}\left\langle A x_{n}, J\left(x_{n}-P_{0} x_{n}\right)\right\rangle+K\left(\left\|x_{n}-P_{0} x_{n}\right\|+t_{n}\left\|A x_{n}\right\|\right. \\
& +C / 2) \rho_{X}\left(t_{n}\left\|A x_{n}\right\|\right) \\
\leqq & \left\|x_{n}-P_{0} x_{n}\right\|^{2}-2 t_{n} \psi\left(\left\|x_{n}-P_{0} x_{n}\right\|\right)\left\|A x_{n}\right\|+K\left(2 \mu\left\|A x_{0}\right\|+T\left(x_{0}\right) M\left(x_{0}\right)\right. \\
& +C / 2) \rho_{X}\left(t_{n}\left\|A x_{n}\right\|\right) \\
\leqq & \left\|x_{n}-P_{0} x_{n}\right\|^{2}-2 t_{n} \psi\left(\left\|x_{n}-P_{0} x_{n}\right\|\right)\left\|A x_{n}\right\|+K\left(3 \mu\left\|A x_{0}\right\|+C / 2\right) \rho_{X}\left(t_{n} M\left(x_{0}\right)\right)
\end{aligned}
$$

If, furthermore, in this case $\left\|A x_{n}\right\|>\left\|A x_{0}\right\|$, then (3.8)-(3.10) yield

$$
\begin{aligned}
2 t_{n} \psi\left(\left\|x_{n}-P_{0} x_{n}\right\|\right)\left\|A x_{n}\right\| & \geqq 2 t_{n} \psi\left(\mu\left\|A x_{0}\right\|\right)\left\|A x_{0}\right\| \\
& \geqq K\left(3 \mu\left\|A x_{0}\right\|+C / 2\right) \rho_{X}\left(t_{n} M\left(x_{0}\right)\right) .
\end{aligned}
$$

From (3.12), it follows that

$$
\left\|x_{n+1}-P_{0} x_{n+1}\right\| \leqq\left\|x_{n}-P_{0} x_{n}\right\| .
$$

If $\left\|A x_{n}\right\| \leqq\left\|A x_{0}\right\|$ in this case (obviously, we can assume $A x_{n} \neq 0$ without loss of generality), then by using the property (p3) of the modulus of smoothness and by (3.7)(3.10), we have

$$
\begin{aligned}
\frac{\rho_{X}\left(t_{n}\left\|A x_{n}\right\|\right)}{t_{n}\left\|A x_{n}\right\|} & \leqq \frac{\rho_{X}\left(t_{n} M\left(x_{0}\right)\right)}{t_{n} M\left(x_{0}\right)} \\
& \leqq \frac{1}{M\left(x_{0}\right)} \frac{2 \psi\left(\mu\left\|A x_{0}\right\|\right)\left\|A x_{0}\right\|}{K\left(3 \mu\left\|A x_{0}\right\|+C / 2\right)} \leqq \frac{2 \psi\left(\mu\left\|A x_{0}\right\|\right)}{K\left(3 \mu\left\|A x_{0}\right\|+C / 2\right)}
\end{aligned}
$$

which yields from (3.12) also that

$$
\begin{aligned}
\left\|x_{n+1}-P_{0} x_{n+1}\right\|^{2} \leqq & \left\|x_{n}-P_{0} x_{n}\right\|^{2}-t_{n}\left\|A x_{n}\right\|\left[2 \psi\left(\mu\left\|A x_{0}\right\|\right)\right. \\
& \left.-K\left(3 \mu\left\|A x_{0}\right\|+C / 2\right) \rho_{X}\left(t_{n}\left\|A x_{n}\right\|\right) /\left(t_{n}\left\|A x_{n}\right\|\right)\right] \\
\leqq & \left\|x_{n}-P_{0} x_{n}\right\|^{2} .
\end{aligned}
$$

This implies that in this case we always have $\left\|x_{n+1}-P_{0} x_{n+1}\right\| \leqq\left\|x_{n}-P_{0} x_{n}\right\| \leqq 2 \mu\left\|A x_{0}\right\|$. completing the proof of Step 1.

Step 2. Show that $\left\{x_{n}\right\}$ is also bounded. For this purpose, we take an $x^{*} \in N(A)$ arbitrarily and let $d_{n}=\max \left\{\left\|x_{n}-x^{*}\right\|, K_{3}\right\}$ where $K_{3}=\max \left\{T\left(x_{0}\right) M\left(x_{0}\right), C / 4\right\}$ and $K_{2}=\max \left\{B M^{2}\left(x_{0}\right), 1\right\}$, where $B, C$ are the constants specified by property (p4) of the modulus of smoothness and Lemma 1 respectively. From Lemma 1 and (1.1), we then have 


$$
\begin{aligned}
&\left\|x_{n+1}-x^{*}\right\|^{2}=\left\|x_{n}-x^{*}-t_{n} A x_{n}\right\|^{2} \\
& \leqq\left\|x_{n}-x^{*}\right\|^{2}-2 t_{n}\left\langle A x_{n}, J\left(x_{n}-x^{*}\right)\right\rangle+K\left(\left\|x_{n}-x^{*}\right\|\right. \\
&\left.\quad+t_{n}\left\|A x_{n}\right\|, C / 2\right) \rho_{X}\left(t_{n}\left\|A x_{n}\right\|\right) \\
& \leqq\left.\left\|x_{n}-x^{*}\right\|^{2}+K\left(\left\|x_{n}-x^{*}\right\|+T\left(x_{0}\right) M\left(x_{0}\right), C / 2\right) \rho_{X}\left(t_{n}\left\|A x_{n}\right\|\right)\right) .
\end{aligned}
$$

Noting that the properties (p1)-(p4) of the modulus of smoothness yield $\rho_{X}\left(t_{n}\left\|A x_{n}\right\|\right) \leqq$ $\rho_{X}\left(t_{n} M\left(x_{0}\right)\right), \quad \rho_{X}\left(t_{n} M\left(x_{0}\right)\right) \leqq \rho_{X}\left(t_{n}\right)$ if $M\left(x_{0}\right) \leqq 1$ and $\rho_{X}\left(t_{n} M\left(x_{0}\right)\right) \leqq B M^{2}\left(x_{0}\right) \rho_{X}\left(t_{0}\right)$ if $M\left(x_{0}\right)>1$, we obtain

$$
\begin{aligned}
\left\|x_{n+1}-x^{*}\right\|^{2} \leqq & \left\|x_{n}-x^{*}\right\|^{2}+K K_{2} \max \left\{2 \operatorname { m a x } \left\{\left\|x_{n}-x^{*}\right\|,\right.\right. \\
& \left.\left.T\left(x_{0}\right) M\left(x_{0}\right)\right\}, \dot{C} / 2\right\} \rho_{X}\left(t_{n}\right) \\
\leqq & \left\|x_{n}-x^{*}\right\|+2 K K_{2} \max \left\{\left\|x_{n}-x^{*}\right\|, K_{3}\right\} \rho_{x}\left(t_{n}\right) .
\end{aligned}
$$

This implies $d_{n+1}^{2} \leqq d_{n}^{2}+2 K K_{2} d_{n} \rho_{X}\left(t_{n}\right)$ and furthermore

$$
d_{n+1}<d_{n}+K K_{2} \rho_{x}\left(t_{n}\right)
$$

Since $\left\{\rho_{x}\left(t_{n}\right)\right\} \in l^{1}$, the convergence of $\left\{d_{n}\right\}$ as $n \rightarrow \infty$ (and in particular, the boundedness of $\left.\left\{\left\|x_{n}-x^{*}\right\|\right\}\right)$ then follows, which in turn, from (3.13), implies the convergence of the sequence $\left\{\left\|x_{n}-x^{*}\right\|\right\}$.

Step 3. Demonstrate that the sequence $\left\{x_{n}\right\}$ converges hyperstrongly to an $x^{*} \in N(A)$. We notice first that a similar reasoning to that in Step 2 implies the existence of the limit $l=\lim _{n \rightarrow \infty}\left\|x_{n}-P_{0} x_{n}\right\|$. If $l>0$ and $\left\{\left\|x_{n+1}-x_{n}\right\|\right) \notin l^{1}$, then, by letting $N_{1}$ be a positive integer such that $\left\|x_{n}-P_{0} x_{n}\right\| \geqq l / 2$ whenever $n \geqq N_{1}$ and $M=K\left[3 \mu\left\|A x_{0}\right\|+C / 2\right]$, we obtain from (3.12) that

$$
\begin{aligned}
\left\|x_{n+1}-P_{0} x_{n+1}\right\|^{2} & \leqq\left\|x_{n}-P_{0} x_{n}\right\|^{2}-2 t_{n} \psi\left(\left\|x_{n}-P_{0} x_{n}\right\|\right)\left\|A x_{n}\right\|+M \rho_{X}\left(t_{n}\left\|A x_{n}\right\|\right) \\
& \leqq\left\|x_{n}-P_{0} x_{n}\right\|-2 t_{n} \psi(l / 2)\left\|A x_{n}\right\|+M \rho_{X}\left(t_{n}\left\|A x_{n}\right\|\right) \\
& \leqq\left\|x_{n}-P_{0} x_{n}\right\|-t_{n}\left\|A x_{n}\right\|\left\{2 \psi(l / 2)-M \rho_{X}\left(t_{n}\left\|A x_{n}\right\|\right) / t_{n}\left\|A x_{n}\right\|\right\}
\end{aligned}
$$

for any $n>N_{1}$. Because $\sup \left\{\left\|A x_{n}\right\|: n \geqq 0\right\} \leqq M\left(x_{0}\right)<+\infty$, the uniform smoothness of $X$ implies that there is an integer $N_{2}>N_{1}$ such that

$$
2 \psi(l / 2)-M \rho_{X}\left(t_{n}\left\|A x_{n}\right\|\right) / t_{n}\left\|A x_{n}\right\| \geqq \psi(l / 2)>0, \quad \forall n \geqq N_{2}
$$

which yields from (3.14) and (1.1)

$$
\left\|x_{n+1}-P_{0} x_{n+1}\right\|^{2} \leqq\left\|x_{n}-P_{0} x_{n}\right\|^{2}-\psi(l / 2)\left\|x_{n+1}-x_{n}\right\|, \quad \forall n \geqq N_{2} .
$$


This clearly implies $\left\{\left\|x_{n+1}-x_{n}\right\|\right\} \in l^{1}$, contradicting the assumption.

Thus two possible cases must occur: either $\lim _{n \rightarrow \infty}\left\|x_{n}-P_{0} x_{n}\right\|=0$ or $\left\{\left\|x_{n+1}-x_{n}\right\|\right\} \in l^{1}$. In the first case, just as in the reasoning leading to (3.12) we can find

$$
\left\|x_{n}-P_{0} x_{m}\right\|^{2} \leqq\left\|x_{m}-P_{0} x_{m}\right\|^{2}+M_{1} \sum_{j=m}^{n-1} \rho_{X}\left(t_{j} M\left(x_{0}\right)\right) \quad \forall n>m>0
$$

where $M_{1}=K \max \left\{\left\|x_{n}-x_{m}\right\|+\left\|x_{m}-P_{0} x_{m}\right\|+\mu\left\|A x_{0}\right\|+C / 2: n, m \geqq 0\right\}<+\infty$. This gives

$$
\begin{aligned}
\left\|x_{n}-x_{m}\right\| \leqq & \left\|x_{n}-P_{0} x_{m}\right\|+\left\|x_{m}-P_{0} x_{m}\right\| \\
\leqq & {\left[\left\|x_{m}-P_{0} x_{m}\right\|^{2}+M_{1} \sum_{j=m}^{n-1} \rho_{X}\left(t_{j} M\left(x_{0}\right)\right)\right]^{1 / 2}+\left\|x_{m}-P_{0} x_{m}\right\| } \\
& \rightarrow 0 \quad(n, m \rightarrow \infty)
\end{aligned}
$$

which shows that $\left\{x_{n}\right\}$ is a Cauchy sequence; in the second case, $\left\{x_{n}\right\}$ certainly is a Cauchy sequence, we then conclude that the limit $x^{*}=\lim _{n \rightarrow \infty} x_{n}$ exists. In the first case, one sees

$$
\left\|x^{*}-P_{0} x^{*}\right\| \leqq\left\|x^{*}-P_{0} x_{n}\right\| \leqq\left\|x^{*}-x_{n}\right\|+\left\|x_{n}-P_{0} x_{n}\right\| \rightarrow 0 \quad(n \rightarrow \infty)
$$

and hence $x^{*} \in N(A)$. In the second case, we have liminf ${ }_{n \rightarrow \infty}\left\|A x_{n}\right\| \rightarrow 0$ hence the demi-closedness of $A$ implies $x^{*} \in N(A)$. This completes the proof of Theorem 1 .

Remark 1. From the proof of Theorem 1, one easily sees that the assumptions of uniform smoothness of $X$ and (3.1) are in fact not required for the necessity of the theorem while the assumption that $A$ satisfies the condition $(J)$ is not required for the sufficiency of the theorem.

Remark 2. We observe that if $A$ is bounded then the assumption (3.1) is satisfied either when $N_{0}(A)$ is bounded, or instead of (3.2) we take $t_{n}=t_{n} / \bar{M}\left(x_{0}\right)$, with $\left\{\bar{t}_{n}\right\}$ being an arbitrary positive sequence satisfying $\left\{\rho_{X}\left(t_{n}\right)\right\} \in l^{1}$ and, for a fixed $x^{*} \in N(A)$, $\bar{M}\left(x_{0}\right)=\sup \left\{\|A x\|: \max \left\{\left\|x-x^{*}\right\|, \bar{K}_{3}\right\} \leqq \max \left\{\left\|x_{0}-x^{*}\right\|, \bar{K}_{3}\right\}+K \sum_{(n)} \rho_{X}\left(t_{n}\right)\right\}$ with $\bar{K}_{3}=$ $\max \left\{\sup \left\{t_{n}\right\}, C / 4\right\}$. For the first situation this claim is straightforward and, for the second case, this can be justified by showing by induction that

$$
\begin{aligned}
\max \left\{\left\|x_{n+1}-x^{*}\right\|, \bar{K}_{3}\right\} & \leqq \max \left\{\left\|x_{n}-x^{*}\right\|, \bar{K}_{3}\right\}+K \rho_{X}\left(\bar{t}_{n}\right) \\
& \leqq \max \left\{\left\{\left\|x_{0}-x^{*}\right\|, \bar{K}_{3}\right\}+K \sum_{j=0}^{n} \rho_{X}\left(t_{j}\right)\right\}
\end{aligned}
$$

(the argument similar to that used in the step 2 of the proof). Additionally, we observe that the demi-closedness of $A$ is naturally satisfied if $A$ is $m$-accretive ([3]). 
Remark 3. In developing their general convergence principle, R. E. Bruck and S. Reich in [7] introduced the following condition (which will be called as the BruckReich condition below): Let $(f, F)$ be an admissible pair (namely, $D(f) \subset D(F) \subset X$, $f: D(f) \rightarrow R^{+}, F: D(F) \rightarrow X^{*}$ and $(f, F)$ satisfies either $f(x+y) \geqq f(x)+\langle y, F(x)\rangle-b(x, y)$ $\|y\|$ or $f(x+y) \leqq f(x)+\langle y, F(x)\rangle+b(x, y)\|y\|$ for all appropriate $x$ and $y$, where $0 \leqq b(x, y) \rightarrow 0$ as $y \rightarrow 0$ uniformly for bounded $x)$. A set-valued mapping $A: D(A) \rightarrow 2^{x}$ is said to satisfy the Bruck-Reich condition if, for each $K>0$, there is an increasing function $g: R^{+} \rightarrow R^{+}$such that $g(r)>0$ for $r>0$, and

$$
\langle y, F(x)\rangle \geqq g(f(x))\|y\|, \quad \forall x \in D(A), y \in A x,\|x\| \leqq K,\|y\| \leqq K .
$$

In particular, if we take $f(x)=\frac{1}{2}\left\|x-P_{0} x\right\|^{2}, F(x)=J_{0}\left(x-P_{0} x\right)$ and let $A$ be singlevalued, then (3.15) leads to the following

$$
\left\langle A x, J_{0}\left(x-P_{0} x\right)\right\rangle \geqq g\left(\frac{1}{2}\left\|x-P_{0} x\right\|\right)\|A x\|, \quad \forall x \in D(A),\|x\| \leqq K,\|A x\| \leqq K .
$$

If, furthermore, we take $x \in\left\{x_{n}\right\}$ in this inequality, where $\left\{x_{n}\right\}$ is the sequence defined by the process (1.1), then (3.16) is nothing else but just (3.3) in Theorem 1. This shows that, in practical applications, it is reasonable to believe that (3.16) actually is a necessary and sufficient condition for strong convergence of the process (1.1) starting from every $x_{0} \in D(A)$. Thus, it is seen that Theorem 1 here generalizes all of the corresponding results in [7], [10], [11] and [16].

\section{The nonlinear semigroup of contractions}

Let $A: D(A) \rightarrow X$ be an accretive operator which satisfies the range condition $\operatorname{cl} D(A) \subset$ $\bigcap\{R(I+t A): t>0\}$. Then it is known that

$$
S(t) x=\lim _{n \rightarrow \infty}\left(I+\frac{t}{n} A\right)^{-n} x
$$

exists for each $x \in \operatorname{cl}(D(A)$ and defines a semigroup of nonlinear contractions $S: c l D(A) \times R^{+} \rightarrow \operatorname{cl} D(A)$.

If, furthermore, $X$ is reflexive and $A$ is closed, it is also known that $S(t) x_{0}$ is the unique strong solution of the evolution equation (1.2) for any $x_{0} \in D(A)$ (cf. [3, p. 118]).

Theorem 2. Let $X$ be a reflexive and strongly smooth Banach space and $A$ be $a$ demi-closed accretive operator which satisfies the condition $(J)$ and the range condition. Then, for any $x_{0} \in D(A), S(t) x_{0}=u(t)$ converges hyperstrongly to an $x^{*}$ in $N(A)$ if and only if there is a nondecreasing function $\varphi: R^{+} \rightarrow R^{+}$such that

$$
\left\langle A u(t), J\left(u(t)-P_{0} u(t)\right)\right\rangle \geqq \varphi\left(\left\|u(t)-P_{0} u(t)\right\|\right)\|A u(t)\|, \quad \forall a . e . t \geqq 0,
$$

where $\varphi(0)=0, \varphi(t)$ is strictly increasing in $t \geqq t^{*}$ with $t^{*}$ given by 


$$
t^{*}=\left\{\begin{array}{l}
\lim _{t \rightarrow \infty}\left\|u(t)-P_{0} u(t)\right\|, \text { if }\left\|\frac{d u}{d t}(t)\right\| \in L^{1}(0, \infty) \\
0, \text { otherwise. }
\end{array}\right.
$$

Proof. For any $t>s$, observe that the contraction property of $S$ implies

$$
\begin{aligned}
& \left\|S(t) x_{0}-P_{0} S(t) x_{0}\right\| \leqq\left\|S(t) x_{0}-P_{0} S(s) x_{0}\right\| \\
& \quad=\left\|S(t-s) S(s) x_{0}-P_{0} S(s) x_{0}\right\|=\left\|S(t-s) S(s) x_{0}-S(t-s) P_{0} S(s) x_{0}\right\| \\
& \quad \leqq\left\|S(s) x_{0}-P_{0} S(s) x_{0}\right\| .
\end{aligned}
$$

It follows that the function $g(t)=\left\|S(t) x-P_{0} S(t) x\right\|=\left\|u(t)-P_{0} u(t)\right\|$ is nonincreasing and therefore we can write $m=\lim _{t \rightarrow \infty} g(t)$. Furthermore we easily find (e.g., see [10, Theorem 1])

$$
\left\langle A u(t), J\left(u(t)-P_{0} u(t)\right)\right\rangle \leqq \frac{1}{2} \frac{d}{d t} g^{2}(t), \text { a.e.t }>0
$$

" $\Rightarrow$ ". Similar to the case in Theorem 1 , by letting $M=\sup \{g(t): t \geqq 0\}, N=$ $\sup \{\|A u(t)\|: u(t) \in D(A)\}, C_{t}=\left\{s \in R^{+}: g(s) \geqq t, u(t) \in D(A)\right\}$ and $f(t)=\inf \{\langle A u(s), J(u(s)-$ $\left.\left.\left.P_{0} u(s)\right)\right\rangle / N: s \in C_{t}\right\}$, we only need to show $f(t)>0$ for any $t \in(m, M)$ under the assumptions that $M>m, m>0$ and $N>0$. Again assume to the contrary, namely, there is a $t_{0} \in(m, M)$ such that $f\left(t_{0}\right)=0$. Then for a subsequence $\left\{t_{i}\right\} \subset C_{t_{0}}$, which is nonempty because $u(t) \in D(A)$, a.e.t $>0$, we have

$$
g\left(t_{i}\right) \geqq t_{0}>m
$$

and

$$
\left\langle A u\left(t_{i}\right), J\left(u\left(t_{i}\right)-P_{0} u\left(t_{i}\right)\right)\right\rangle \rightarrow 0 \quad(i \rightarrow \infty) .
$$

Now we need to distinguish two possible cases:

Case 1. If $\left\{t_{i}\right\}$ contains an infinite subsequence which tends to infinity, then by convergence of $g(t)$ as $t$ goes to infinity we deduce that $u\left(t_{i}\right)$ is finitely circulative and consequently we come to a contradiction just as in the proof of Theorem 1.

Case 2. If $\left\{t_{i}\right\}$ is contained in an interval, say, $[0, T]$, in this case we can assume without loss of generality that $t_{i}$ tends to a $t^{*} \in[0, T]$. Since $A$ is semi-closed, $g(t)$ is nondecreasing and the strong smoothness of $X$ implies that $J$ is continuous from the strong topology of $X$ to the strong topology of $X^{*}$. (4.3) and (4.4) then give

$$
g\left(t^{*}\right) \geqq t_{0}>m
$$

and $\left\langle A u\left(t^{*}\right), J\left(u\left(t^{*}\right)-P_{0} u\left(t^{*}\right)\right)\right\rangle=0$, from which $u\left(t^{*}\right) \in N(A)$ clearly follows (for, $A$ satisfies the condition $(J))$. Furthermore, we observe that for any $t>t^{*}$, 


$$
u(t)=S(t) x_{0}=S\left(t-t^{*}\right) S\left(t^{*}\right) x_{0}=S\left(t-t^{*}\right) u\left(t^{*}\right)=u\left(t^{*}\right)
$$

Hence $u(t) \rightarrow u\left(t^{*}\right)$ as $t \rightarrow \infty$, which yields $g\left(t^{*}\right)=\left\|u\left(t^{*}\right)-P_{0} u\left(t^{*}\right)\right\|=\lim _{t \rightarrow \infty} g(t)=m$. This contradicts (4.5) and hence completes the proof of the necessity.

“”. (4.1) together with (4.2) now implies

$$
\varphi(g(t))\|A u(t)\| \leqq\left\langle A u(t), J\left(u(t)-P_{0} u(t)\right)\right\rangle \leqq-\frac{1}{2} \frac{d}{d t} g^{2}(t)
$$

and hence

$$
\int_{s}^{t} \varphi(g(\mu))\|A u(\mu)\| d \mu \leqq \frac{1}{2}\left[g^{2}(s)-g^{2}(t)\right]
$$

for any $t \geqq s>0$. If $m \neq 0$ and $\|A u(t)\| \notin L^{1}(0, \infty)$, then there is a $T_{0}>0$ such that $g(t) \geqq m / 2, \forall t>T_{0}$, which yields

$$
\varphi(m / 2) \int_{s}^{t}\|A u(\mu)\| d \mu \leqq \frac{1}{2}\left[g^{2}(s)-g^{2}(t)\right], \quad \forall T_{0} \leqq s<t
$$

By the definition of $t^{*}, \varphi(t)$ in this case is strictly increasing in $t>0$, so $\varphi(m / 2)>0$. From (4.7) it then follows that $\|A u(t)\| \in L^{1}(0, \infty)$, yielding an obvious contradiction. Accordingly we have that either $m=0$ or $\|A u(t)\| \in L^{1}(0, \infty)$.

If $m=0$, then for any $h \geqq 0$

$$
\begin{aligned}
\|u(t)-u(t+h)\| & \leqq\left\|u(t)-P_{0} u(t)\right\|+\left\|u(t+h)-P_{0} u(t)\right\| \\
& \leqq\left\|u(t)-P_{0} u(t)\right\|+\left\|S(h) u(t)-P_{0} u(t)\right\| \leqq 2 g(t) \rightarrow 0
\end{aligned}
$$

as $t \rightarrow \infty$ and hence $x^{*}=\lim _{t \rightarrow \infty} u(t)$ exists. From the above inequality clearly, $\left\|u(t)-x^{*}\right\|$ $\leqq 2 g(t)$. This gives

$$
\left\|x^{*}-P_{0} x^{*}\right\| \leqq\left\|x^{*}-P_{0} u(t)\right\| \leqq\left\|x^{*}-u(t)\right\|+\left\|u(t)-P_{0} u(t)\right\| \leqq 3 g(t)
$$

from which $x^{*} \in N(A)$ follows. Suppose $m \neq 0$ but

$$
\|A u(t)\|=\left\|\frac{d u}{d t}(t)\right\| \in L^{1}(0, \infty) .
$$

Then $u(t)$ is convergent, and in particular, if $y^{*}=\lim _{n \rightarrow \infty} u(t)$, then $A y^{*}=0$ because of the semiclosedness of $A$. This completes the proof of Theorem 2.

Remark 4. Theorem 2 extends [7, Th. 3.3] and the corresponding results in [10] and [11]. 


\section{REFERENCES}

1. F. E. Browder, Nonlinear mappings of nonexpansive and accretive type in Banach spaces, Bull. Amer. Math. Soc. 73 (1967), 875-882.

2. F. E. Browder, Nonlinear operators and nonlinear equations of evolutioin in Banach spaces, (Proc. Sympos. Pure Math., Vol. 18, Part 2, AMS, Providence, R.I., 1976).

3. V. Barbu, Nonlinear Semigroups and Differential Equations in Banach Spaces (Noordhoff, Leiden, 1976).

4. R. E. Bruck, Asymptotic convergence of nonlinear contraction semigroups in Hilbert space, J. Funct. Anal. 18 (1975), 15-26.

5. R. E. BRUCK, An iterative solution of a variational inequality for certain monotone operators in Hilbert space, Bull. Amer. Math. Soc. 81 (1975), 890-892. Corrigendum, Bull. Amer. Math. Soc. 82 (1976), 353.

6. R. E. BRUCK, Asymptotic behavior of nonexpansive mappings, Contemp. Math. 18 (1983), $1-47$.

7. R. E. Bruck and S. ReICH, A general convergence principle in nonlinear functional analysis, Nonlinear Anal. 4 (1980), 939-950.

8. M. G. Crandall and A. Pazy, On the range of accretive operators, Israel J. Math. 27 (1977), 235-246.

9. J. Lindenstrauss and L. Tzafriri, Classical Banach Space II-Function Spaces (SpringerVerlag, Berlin, 1979).

10. O. Nevanlinna and S. Reich, Strong convergence of contraction semigroups and of iterative methods for accretive operators in Banach spaces, Israel J. Math. 32 (1979), 44-58.

11. A. PazY, Semigroups of nonlinear contraction and their asymptotic behavior, in Nonlinear Analysis and Mechanics (Heriot-Watt Symposium, Vol. III, Research Notes in Math., 30, Pitman, London, 1979).

12. S. ReICH, Weak convergence theorems for nonexpansive mappings in Banach spaces, $J$. Math. Anal. Appl. 67 (1979), 274-276.

13. S. ReICH, Asymptotic behavior of semigroups of nonlinear contraction in Banach space, $J$. Math. Anal. Appl. 53 (1976), 277-290.

14. S. ReICH, On the asymptotic behavior of nonlinear semigroups and the range of accretive operators, J. Math. Anal. Appl. 79 (1981), 113-126.

15. Z. B. Xu and G. F. RoACH, Characteristic inequalities of uniform convex and uniform smooth Banach spaces, J. Math. Anal. Appl. 157 (1991), 189-210.

16. Z. B. Xu and G. F. RoACH, A necessary and sufficient condition for convergence of steepest descent approximation to accretive operator equations, J. Math. Anal. Appl. 167 (1992), 340-354.

17. Z. B. XU, B. ZHANG and G. F. RoACH, On the steepest-descent approximation to solutions of nonlinear strongly accretive operator equations, J. Comput. Math. Supp. (1992), 173-182.

\section{Zong-Ben Xu ANd Yao-Lin Jiang}

Institute for Computational and Applied Mathematics

XI'AN JIAOTONG UNIVERSITY

XI'AN

China
G. F. ROACH

Department of Mathematics UNIVERSITY OF STRATHCLYDE GLASGOW

SCotland 